\title{
Neuroprotective effect of heat shock protein 60 on matrine-suppressed microglial activation
}

\author{
RUI ZHANG ${ }^{1 *}$, YUNHONG LI $^{1 *}$, XIAOLIN HOU ${ }^{2 *}$, ZHENHUA MIAO $^{1}$ and YIN WANG ${ }^{1}$ \\ ${ }^{1}$ Ningxia Key Laboratory of Cerebrocranial Diseases, Basic Medical School of Ningxia Medical University; \\ ${ }^{2}$ Department of Neurology, General Hospital of Ningxia Medical University, Yinchuan, Ningxia 750004, P.R. China
}

Received June 16, 2016; Accepted April 21, 2017

DOI: $10.3892 /$ etm.2017.4691

\begin{abstract}
Matrine (MT) is the primary active alkaloid separated from members of the Sophora genus. Previous studies have reported that MT has anti-inflammatory effects in the central nervous system (CNS). However, the underlying molecular mechanism of the neuroprotective effect of MT remains unclear, particularly the role of heat shock protein 60 (HSP60). Microglia are macrophages in the CNS that serve an essential role in the innate immune system by producing various proinflammatory and neurotoxic factors. In addition, HSP60 is released by activated microglia causing an autoimmune response. The present study aimed to investigate whether MT could inhibit the activation of microglia via suppressing the HSP60 signaling pathway. The results demonstrated that the expression and release of HSP60 in LPS-activated BV2 microglial cells was significantly decreased by MT treatment. Extracellular HSP60 is a ligand of toll like receptor 4 (TLR-4); thus, it was hypothesized that secreted HSP60 could bind to TLR- 4 on microglia and activate the TLR-4 signaling pathway. As expected, western blotting and ELISA results revealed that MT significantly inhibited the LPS-induced increase in TLR-4, myeloid differentiation primary response protein MyD88, caspase-3 and tumor necrosis factor- $\alpha$. In conclusion, the results of the present study provide a novel direction for the prevention and treatment of neurodegenerative diseases characterized by microglial activation.
\end{abstract}

\section{Introduction}

Microglia are the resident macrophages of the central nervous system (CNS). Accumulating evidence has demonstrated that

Correspondence to: Professor Yin Wang, Ningxia Key Laboratory of Cerebrocranial Diseases, Basic Medical School of Ningxia Medical University, 1160 Shengli Street, Yinchuan, Ningxia 750004, P.R. China

E-mail: yin-wang@hotmail.com

*Contributed equally

Key words: matrine, heat shock protein 60, toll-like receptor 4, BV2 microglial cells the over-activation of microglia, for example, in response to certain environmental toxins and endogenous proteins, contributes to the progression of several neurodegenerative diseases, including Alzheimer's disease, Parkinson's disease and multiple sclerosis (MS) (1). Previous studies have indicated that neurodegenerative diseases are associated with the secretion of various proinflammatory and cytotoxic factors by activated microglia in the brain (2-5). Therefore, inhibiting the activation of microglia is an important strategy for the prevention of neurodegeneration.

Microglia can be activated by lipopolysaccharide (LPS), and serve a role in the innate and adaptive immune responses through the production of proinflammatory mediators, including myeloid differentiation primary response protein MyD88 (MyD88), nuclear factor- $\kappa \mathrm{B}$, caspase-3 and heat shock protein 60 (HSP60) $(6,7)$. It has been demonstrated that HSP60 is highly expressed by activated microglia, and that the extracellular release of HSP60 increases the production of other proinflammatory factors through binding to toll-like receptor 4 (TLR-4) and stimulating neuronal cell death $(8,9)$. Thus, the regulation of HSP60 production is a potential therapeutic option for the treatment of neurodegenerative disorders.

Matrine (MT), the major active component of members of the Sophora genus, is used to treat inflammatory diseases and cancer in traditional Chinese medicine $(10,11)$. Kan et al (12) demonstrated that MT could attenuate the severity of experimental autoimmune encephalomyelitis through reducing levels of chemokine ligand 2 and C-X-C motif chemokine 10, and suggested that MT may be an effective immunomodulatory therapeutic approach for MS through inhibiting immune cell recruitment mechanisms. MT was also identified to effectively protect neuronal axons from CNS inflammation-induced damage by inhibiting risk factors, including $\beta$-secretase 1 , and upregulating neuroprotective factors, including brain-derived neurotrophic factor (13). However, the cellular and molecular mechanisms underlying the anti-inflammatory activity of MT on microglia remain unclear (14). The present study aimed to investigate the neuroprotective effects of MT and determine whether HSP60 was associated with these effects through inhibiting microglial activation. The results demonstrated that MT could inhibit the expression of HSP60, TLR-4, heat shock factor 1 (HSF-1), caspase-3 and MyD88 to prevent neuronal injury in LPS-treated BV2 mouse microglial cells. These 
results suggest that MT prevents microglial activation via inhibiting the HSP60/TLR-4/MyD88 signaling pathway.

\section{Materials and methods}

Chemicals. BV2 mouse microglial cells were purchased from the Cell Bank of the Chinese Academy of Sciences (Shanghai, China). LPS and MT were purchased from Sigma-Aldrich (Merck KGaA, Darmstadt, Germany). The anti- $\beta$-actin (cat. no. TA-09) antibody was purchased from ZSGB-BIO Technology Co., Ltd. (Beijing, China). Antibodies directed against HSP60 (cat. no. ADI-SPA-806-D) and HSF-1 (cat. no. ADI-SPA-950-D) were purchased from Stressgen Biotechnologies (San Diego, CA, USA). Antibodies directed against caspase-3 (cat. no. 9665), MyD88 (cat. no. 4283) and TLR-4 (cat. no. 2219) were obtained from Cell Signaling Technology, Inc. (Danvers, MA, USA). Tumor necrosis factor TNF- $\alpha$ ELISA kit (mouse TNF- $\alpha$ ELISA EMC102a.96) was acquired from Xinbosheng Biotechnology, Inc. (Shenzhen, China), and HSP60 ELISA kit (mouse HSP-60 ELISA kit, E-20344) was purchased from Beijing Chenglin Biotechnology, Inc. (Beijing, China). Dulbecco's modified Eagle's medium (DMEM) and fetal bovine serum (FBS) were obtained from Gibco ${ }^{\circledR}$ (Thermo Fisher Scientific, Inc., Waltham, MA, USA). The Cell Counting Kit-8 (CCK-8) was obtained from BestBio (Shanghai, China). The bicinchoninic acid (BCA) and enhanced chemiluminescence (ECL) kits were acquired from Pierce (Thermo Fisher Scientific, Inc.).

Cell culture. Mouse BV2 microglial cells were maintained in DMEM supplemented with $10 \% \mathrm{FBS}$ at $37^{\circ} \mathrm{C}$ with $5 \% \mathrm{CO}_{2}$ in an incubator. Cells were pretreated with LPS $(1 \mu \mathrm{g} / \mathrm{ml})$ for $30 \mathrm{~min}$ and then incubated with different concentrations (5, 10,20 or $50 \mu \mathrm{g} / \mathrm{ml}$ ) of MT for $24 \mathrm{~h}$ prior to a variety of assays.

Cell viability assay. Cell viability was assayed using the CCK-8 kit. Cells were seeded into 96-well microtiter plates at a density of $5 \times 10^{4}$ cells/well and cultured for $24 \mathrm{~h}$. Subsequently, CCK-8 solution $(10 \mu \mathrm{l})$ was added to each well according to the manufacturer's instructions and the plates were incubated for a further $2 \mathrm{~h}$. The absorbance at $450 \mathrm{~nm}$ was measured using a microplate reader in order to determine cell viability as a percentage of the control (untreated with MT) value.

ELISA for TNF- $\alpha$ and HSP60. Levels of TNF- $\alpha$ and HSP60 released into the culture media were determined using TNF- $\alpha$ and HSP60 ELISA kits according to the manufacturer's protocol. The absorbance was detected at $450 \mathrm{~nm}$ using a microplate reader in order to determine the amount of TNF- $\alpha$ and HSP60.

Western blotting. Cells were washed three times with PBS ( $2 \mathrm{~min}$ for each wash) and lysed $\left(10 \mathrm{~min}, 4^{\circ} \mathrm{C}\right)$ with radioimmunoprecipitation assay buffer. The lysate was centrifuged $\left(1,200 \mathrm{x} \mathrm{g}, 15 \mathrm{~min}, 4^{\circ} \mathrm{C}\right)$ and the supernatant was collected. Protein concentration was measured using the Pierce BCA kit following the manufacturer's protocol. Equal quantities of protein $(10 \mu \mathrm{l})$ were resolved by SDS-PAGE on a $12 \%$ gel for $90 \mathrm{~min}$. The resolved protein was transferred onto a polyvinylidene difluoride membrane, which were blocked

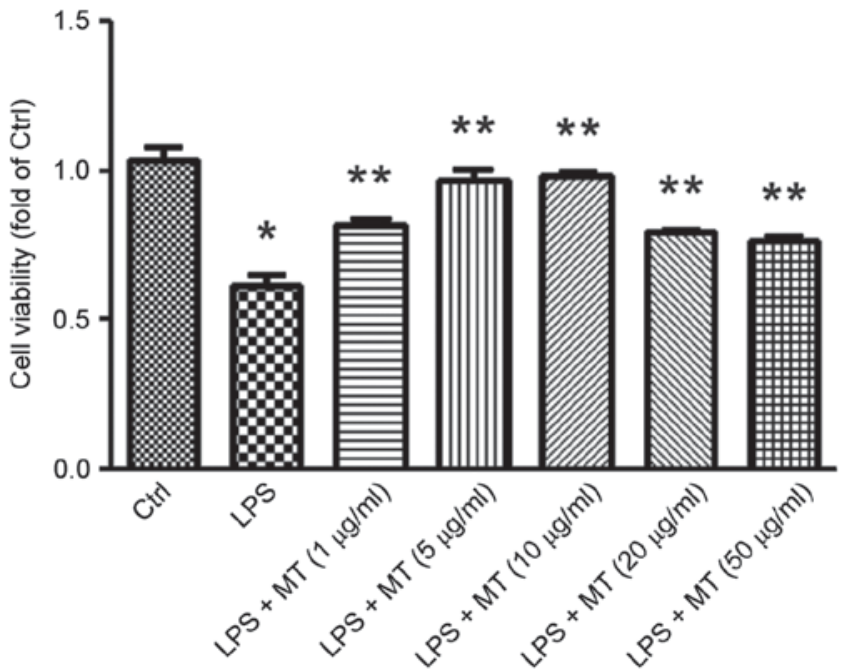

Figure 1. MT increases LPS-treated BV2 cell viability. BV2 microglia cells were stimulated with $1 \mu \mathrm{g} / \mathrm{ml}$ LPS for $30 \mathrm{~min}$, followed by treatment with different concentrations of MT for $24 \mathrm{~h}$ prior to detection of cell viability. ${ }^{*} \mathrm{P}<0.05$ vs. the Ctrl group; ${ }^{* *} \mathrm{P}<0.05$ vs. the LPS group. Ctr, control; LPS, lipopolysaccharide; MT, matrine.

(60 min, room temperature) with 5\% dried milk. Subsequently, the membranes were incubated with primary antibodies in Tris-buffered saline-Tween-20 (TBST) directed against HSP60 (1:1,000 dilution), MYD88 (1:1,000 dilution), HSF-1 (1:1,000 dilution), TLR-4 (1:1,000 dilution), caspase-3 (1:2,000 dilution) or $\beta$-actin (1:1,000 dilution) at $4^{\circ} \mathrm{C}$ overnight. After washing with $\mathrm{PBS}$, the membranes were incubated with anti-mouse (cat. no. ZB-2305; 1:5,000 dilution) or anti-rabbit (cat. no. ZB-2301; 1:5,000 dilution) secondary antibodies diluted in TBST for $1 \mathrm{~h}$ at room temperature. Proteins bands were then visualized using the ECL kit and X-ray films. The results were quantified using Quantity One software, version 4.6.9 (Bio-Rad Laboratories, Inc., Hercules, CA, USA).

Statistical analysis. Results are presented as the mean \pm standard error of the mean of three independent experiments. One-way analysis of the variance followed by a post hoc Student-Newman-Keuls test was used statistically analyze the significance of differences between groups. $\mathrm{P}<0.05$ was considered to indicate a statistically significant difference. SPSS 19.0 software (IBM Corp., Armonk, NY, USA) was used for statistical analysis.

\section{Results}

MT increases LPS-treated BV2 cell viability. The CCK-8 assay was performed to detect the effect of MT on the viability of LPS-stimulated BV2 cells. The results indicated that after treatment with $1 \mu \mathrm{g} / \mathrm{ml}$ LPS for 30 min the viability of BV2 cells significantly decreased compared with the control group ( $\mathrm{P}<0.05$; Fig. 1). However, when LPS treatment was followed by MT treatment $(5,10,20$ or $50 \mu \mathrm{g} / \mathrm{ml})$ for $24 \mathrm{~h} \mathrm{BV} 2$ cell viability was significantly increased compared with the group treated with LPS alone (all $\mathrm{P}<0.05$; Fig. 1 ). BV2 cells treated with 5-10 $\mu \mathrm{g} / \mathrm{ml}$ MT exhibited the optimal increase in viability; thus, $7.5 \mu \mathrm{g} / \mathrm{ml}$ MT was used in subsequent experiments. 
A
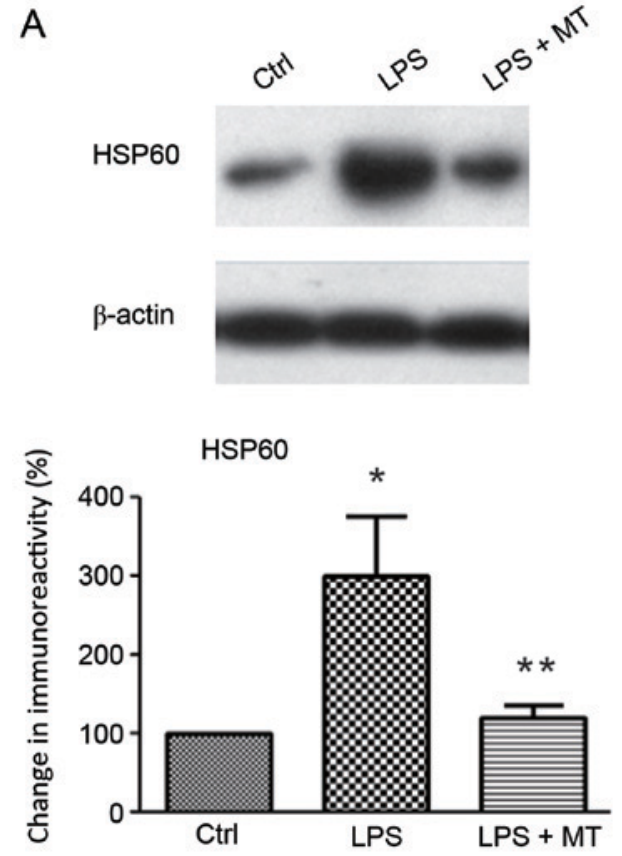

C

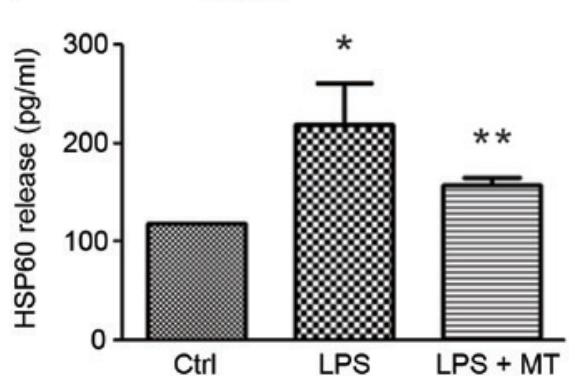

B
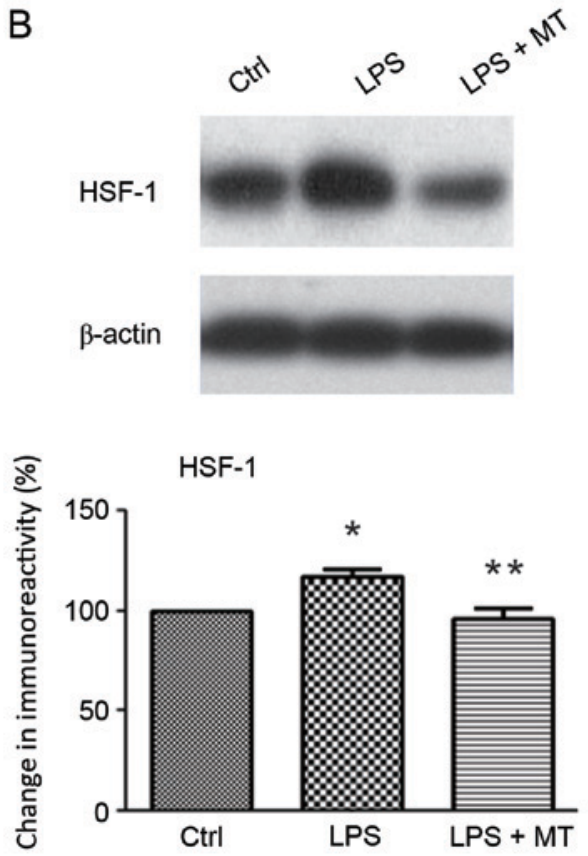

Figure 2. HSP60 expression is inhibited by MT in LPS-stimulated BV2 cells. BV2 microglia cells were stimulated with $1 \mu \mathrm{g} / \mathrm{ml}$ LPS for 30 min, followed by treatment with $7.5 \mu \mathrm{g} / \mathrm{ml}$ MT for $24 \mathrm{~h}$ prior to detection of HSP60 and HSF-1. (A) HSP60 and (B) HSF-1 expression levels were detected via western blotting. (C) HSP60 protein expression was detected via ELISA. 'P<0.05 vs. the Ctrl group; ${ }^{* *} \mathrm{P}<0.05$ vs. the LPS group. Ctrl, control; LPS, lipopolysaccharide; MT, matrine; HSP, heat shock protein; HSF, heat shock factor.

HSP60 expression is inhibited by MT in LPS-stimulated BV2 cells. Western blotting and ELISA were used to investigate the effects of MT on HSP60, and HSF-1 expression (Fig. 2). The results indicated that LPS stimulation significantly increased the level of HSP60 compared with the control group; however, MT treatment following LPS stimulation significantly reduced this increase (both $\mathrm{P}<0.05$; Fig. $2 \mathrm{~A}$ ). Similarly, the ELISA results demonstrated that the release of extracellular HSP60 in LPS-stimulated BV2 cells was significantly inhibited by MT compared with cells treated with LPS alone ( $\mathrm{P}<0.05$; Fig. $2 \mathrm{C}$ ). HSF-1 is a transcription factor that regulates HSP60 expression and release (9). Western blot analysis revealed that the expression of HSF-1 was significantly upregulated by LPS compared with the control group; however, subsequent MT treatment significantly reduced this increase (both $\mathrm{P}<0.05$; Fig. $2 \mathrm{~B}$ ). A previous study reported that HSP60 may translocate extracellularly during cellular stress in order to induce apoptosis (15).

MT decreases the expression of TLR-4, MyD88 and caspase-3 proteins in LPS-stimulated BV2 cells. The expression of proteins in the TLR-4/MyD88 signaling pathway was measured by western blotting after treatment with LPS and MT. The results demonstrated that the expression of TLR-4 was significantly inhibited by MT in LPS-stimulated BV2 cells compared with cells treated with LPS alone ( $<<0.05$; Fig. 3A). MyD88 and caspase- 3 are downstream signaling molecules of TLR-4. Western blot analysis demonstrated that MT significantly suppressed the expression of MyD88 (Fig. 3B) and caspase-3 (Fig. 3C) compared with cells treated with LPS alone (both $\mathrm{P}<0.05$ ). These results indicate that MT exerts its neuroprotective effects through inhibiting the TLR-4/MyD88 signaling pathway, which subsequently inhibits microglial activation.

$M T$ reduces proinflammatory cytokine $T N F-\alpha$ production in LPS-stimulated BV2 cells. To evaluate the effect of MT on the production of inflammatory factors, the level of TNF- $\alpha$ in the culture media was measured using an ELISA. This revealed that MT significantly inhibited the release of TNF- $\alpha$ in LPS-induced BV2 cells compared with cells treated with LPS alone $(\mathrm{P}<0.05$; Fig. 4). This indicates that MT may prevent neuronal cell death by suppressing the production of inflammatory factors. 
A
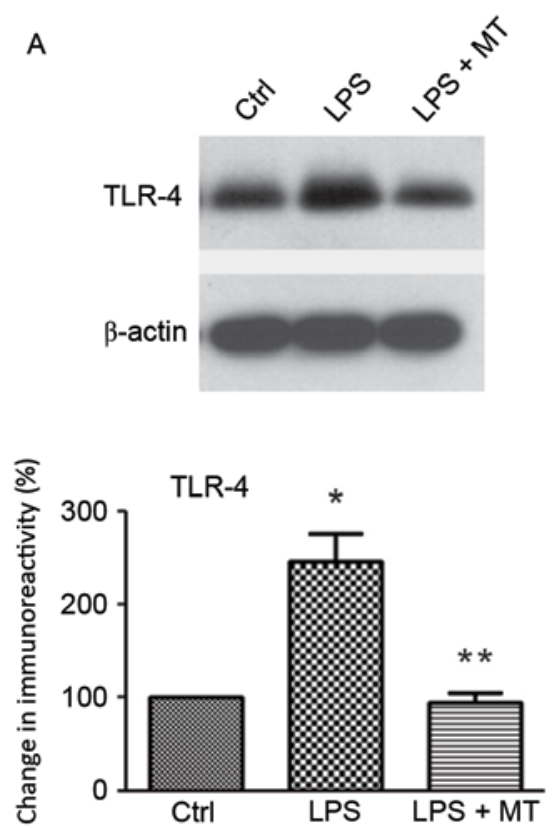

B
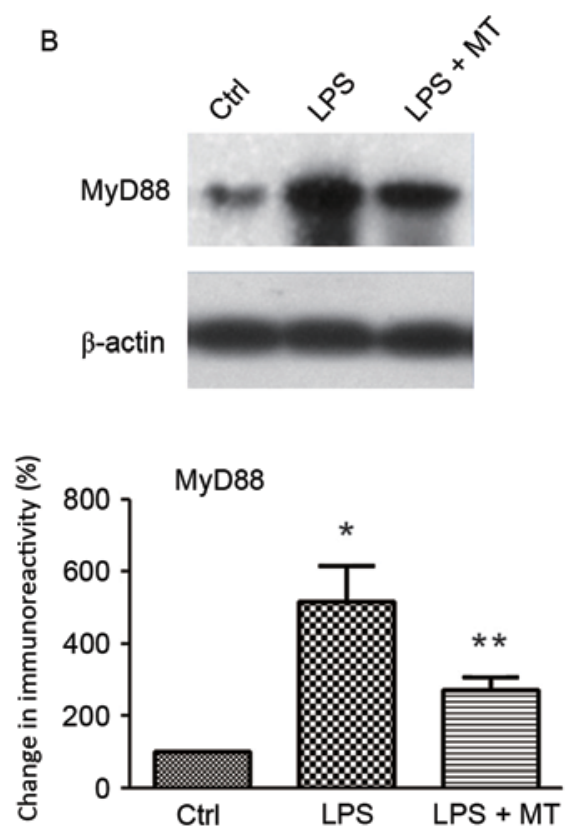

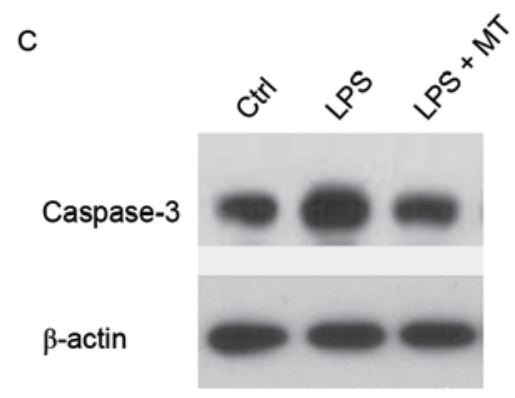

Figure 3. MT decreases the expression of TLR-4, MyD88 and caspase-3 proteins in LPS-stimulated BV2 cells. BV2 microglia cells were stimulated with $1 \mu \mathrm{g}$ with $1 \mu \mathrm{g} / \mathrm{ml}$ LPS for $30 \mathrm{~min}$, followed by treatment with $7.5 \mu \mathrm{g} / \mathrm{ml} \mathrm{MT}$ for $24 \mathrm{~h}$ prior to western blotting for TLR-4, MyD88 and caspase-3. Western blotting of (A) TLR-4, (B) MyD88 and (C) caspase-3. "P<0.05 vs. the Ctrl group, ${ }^{* *} \mathrm{P}<0.05$ vs. the LPS group. Ctrl, control; LPS, lipopolysaccharide; MT, matrine; TLR, toll-like receptor; MyD88, myeloid differentiation primary response protein MyD88.

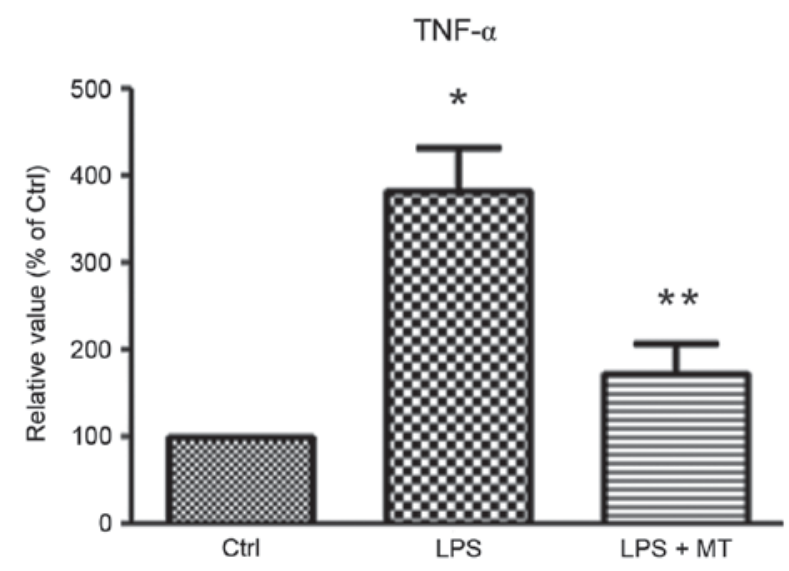

Figure 4. MT reduces proinflammatory cytokine TNF- $\alpha$ production in LPS-stimulated BV2 cells. BV2 microglia cells were stimulated with $1 \mu \mathrm{g} / \mathrm{ml}$ LPS for $30 \mathrm{~min}$, followed by treatment with $7.5 \mu \mathrm{g} / \mathrm{ml} \mathrm{MT}$ for $24 \mathrm{~h}$ prior to ELISA detection of TNF- $\alpha$. " $\mathrm{P}<0.05$ vs. the Ctrl group; ${ }^{* *} \mathrm{P}<0.05$ vs. the LPS group. Ctrl, control; LPS, lipopolysaccharide; MT, matrine; TNF, tumor necrosis factor.

\section{Discussion}

Microglia serve a primary role in the immune response in the CNS, and activated microglia secrete numerous proinflammatory and neurotoxic factors, which are responsible for inflammation-associated and neurodegenerative diseases (16). Inhibiting microglial activation, in order to reduce the production of proinflammatory and neurotoxic factors, may be an effective method for the prevention and treatment of neurodegenerative diseases.

LPS has been demonstrated to induce the activation of microglia (17). Here, $1 \mu \mathrm{g} / \mathrm{ml}$ LPS was used to stimulate microglia BV2 cells for $30 \mathrm{~min}$ in order to establish a microglial activation model. The effect of MT on activated microglia was then investigated via cell viability assays, western blotting and ELISAs. Western blotting results revealed that the expression of HSF-1, MyD88, HSP60 and TLR-4 increased significantly after LPS stimulation, and that MT treatment could significantly reduce this increase. ELISA results demonstrated that MT could significantly reduce the expression of HSP60 and TNF- $\alpha$. These results indicate that MT inhibits microglial activation via inhibiting the HSP60/TLR-4/MyD88 signaling pathway.

MT, an alkaloid with low toxicity, is widely used in clinical treatment, for example, to treat silicosis and prevent liver function damage of anti-tumor drugs $(18,19)$. MT has been demonstrated to exert anti-inflammatory, antitumor, antiarrhythmia, antipyretic, analgesic and anticonvulsant effects in the CNS $(12,13)$. However, it remains unclear whether MT has neuroprotective effects on microglia.

HSP60 is a mitochondrial protein with dual roles. Under normal conditions, HSP60 acts as a molecular chaperone, assisting in polypeptide transposition, folding and assembly. When HSP60 is overexpressed or ectopically expressed under conditions of stress, HSP60 acts as a self-antigen, which is recognized by the immune system and causes an immune response (20). HSP60 also acts as a signal molecule, serving a role in signal transduction (21-23). Activated HSP60 is primarily localized in the plasma membrane or extracellular space, where it exerts a proapoptotic effect by enhancing caspase activation (24). LPS-induced microglia cells are toxic when caspase-3 is active, however activated microglia have been demonstrated not to be toxic to neighboring neurons when caspase- 3 is inhibited $(25,26)$. The transcription factor HSF-1 regulates the expression of HSP60 by binding to its promoter (27). The results of the present study demonstrated that MT significantly decreased the expression and release 
of HSP60, caspase-3 and HSF-1 in LPS-stimulated BV2 microglia cells.

HSP60 is a ligand of TLR-4, which is a component of two signaling pathways, one that is MyD88-dependent and another that is independent of MyD88. MyD88 is a common receptor for all of the known TLRs, excluding TLR-3 (28). The results of the present study identified that the protein expression of TLR-4 and MyD88 significantly increased in LPS-stimulated BV2 cells, and that MT significantly inhibited this increase. Activated microglia cells release a large amount of proinflammatory cytokines, including TNF- $\alpha$ and interleukin $1 \beta$. TNF- $\alpha$ serves important roles in the immune response and apoptosis, and this increased expression of TNF- $\alpha$ is associated with the destruction of dopaminergic neurons (29). ELISA results from the present study demonstrated that TNF- $\alpha$ was released by BV2 cells upon LPS activation, and that this significant increase in extracellular TNF- $\alpha$ could be inhibited by MT.

In conclusion, the results of the present study indicate that MT inhibits the activation of microglia by suppressing the HSP60/TLR-4/MyD88 signaling pathway, and that this inhibited has a neuroprotective and anti-inflammatory effect. Thus, MT is a potential neuroprotective agent. These findings may provide a novel direction for the treatment of neurodegenerative diseases.

\section{Acknowledgements}

The present study was supported by Ningixa 13th Plan of Five-Year Major Scientific Program (grant no. 2016BZ07) and the National Natural Science Foundation of China (grant nos. 31460257, 81460182, 81571098, 31560273 and 31260243).

\section{References}

1. Perry VH, Nicoll JA and Holmes C: Microglia in neurodegenerative disease. Nat Rev Neurol 6: 193-201, 2010.

2. Block ML, Zecca L and Hong JS: Microglia-mediated neurotoxicity: Uncovering the molecular mechanisms. Nature Rev Neurosci 8: 57-69, 2007.

3. Liu B and Hong JS: Role of microglia in inflammation-mediated neurodegenerative disease: Mechanisms and strategies for therapeutic intervention. J Pharmacol Exp Ther 304: 1-7, 2003.

4. Hanisch Uk and Kettenmann H: Microglia: Active sensor and versatile effector cells in the normal and pathologic brain. Nat Neurosci 10: 1387-1394, 2007.

5. Gehrmann J, Matsumoto Y and Kreutzberg GW: Microglia: Intrinsic immuneffector cell of the brain. Brain Res Rev 20: 269-287, 1995.

6. Lynch MA: The multifaceted profile of activated microglia. Mol Neurobiol 40: 139-156, 2009

7. Li YH, Teng P, Wang Y, Zhang YM, Ma CJ and Pu J: Expression and regulation of HSP60 in activated microglia cells. J Ningxia Med Univ 8: 712-714, 2011

8. Zhang D, Sun L, Zhu H, Wang L, Wu W, Xie J and Gu J: Microglial LOX-1 reacts with extracellular HSP60 to bridge neuroinflammation and neurotoxicity. Neurochem Int 61: 1021-1035, 2012.

9. Cheng W, Li Y, Hou X, Zhang N, Ma J, Ding F, Li F, Miao Z, Zhang Y, Qi Q, et al: HSP60 is involved in the neuroprotective effects of naloxone. Mol Med Rep 10: 2172-2176, 2014.

10. Zhao P, Zhou R, Zhu XY, Hao YJ, Li N, Wang J, Niu Y, Sun T, Li YX and Yu JQ: Matrine attenuates focal cerebral ischemic injury by improving antioxidant activity and inhibiting apoptosis in mice. Int J Mol Med 36: 633-644, 2015.
11. Rong B, Zhao C, Gao W and Yang S: Matrine promotes the efficacy and safety of platinum-based doublet chemotherapy for advanced non-small cell lung cancer. Int J Clin Exp Med 8: 14701-14717, 2015.

12. Kan QC, Pan QX, Zhang XJ, Chu YJ, Liu N, Lv P, Zhang GX and Zhu L: Matrine ameliorates experimental autoimmune encephalomyelitis by modulating chemokines and their receptors. Exp Mol Pathol 99: 212-219, 2015.

13. Kan QC, Lv P, Zhang XJ, Xu YM, Zhang GX and Zhu L: Matrine protects neuro-axon from CNS inflammation-induced injury. Exp Mol Pathol 98: 124-130, 2015.

14. Zhang ML, Zhang XJ, Kang J, Zhang HJ, Chen XL, Liu N, Liu SQ, Ma WD, Zhang GX and Zhu L: Matrine promotes NT3 expression in CNS cells in experimental autoimmune encephalomyelitis. Neurosci Lett 649: 100-106, 2017.

15. Chandra D, Choy G and Tang DG: Cytosolic accumulation of HSP60 during apoptosis with or without apparent mitochondrial release: evidence that its pro-apoptotic or pro-survival functions involve differential interactions with caspase-3. J Biol Chem 282: 31289-31301, 2007.

16. Kreutzberg GW: Microglia: A sensor for pathological events in the CNS. Trends Neurosci 19: 312-328, 1996.

17. Teng P, Li Y, Cheng W, Zhou L, Shen Y and Wang Y: Neuroprotective effects Of Lycium barbarum polysaccharides in lipopolysaccharide-induced BV2 microglia cells. Mol Med Rep 7: 1977-1981, 2013.

18. Miao RM, Fang ZH and Yao Y: Therapeutic efficacy of tetrandrine tablets combined with matrine injection in treatment of silicosis. Zhonghua Lao Dong Wei Sheng Zhi Ye Bing Za Zhi 30: 778-780, 2012 (In Chinese).

19. Lao Y: Clinical study of matrine injection on preventing liver function damage of anti-tumor drugs during chemotherapy of breast cancer. Zhong Yao Cai 28: 735-737, 2005 (In Chinese).

20. Quintana FJ and Cohen IR: HSP60 speaks to the immune system in many voices. Novartis Found symp 291: 101-114, 2008.

21. Lehnardt S, Schott E, Trimbuch T, Laubisch D, Krueger C, Wulczyn G, Nitsch R and Werber JR: A vicious cycle involving release of heat shock protein 60 from injured cells and activation of toll-like receptor 4 mediates neurodegeneration in the CNS J Neurosci 28: 2320-2331, 2008.

22. Hansen JJ, Bross P, Westergaard M, Nielsen MN, Eiberg H, Børglum AD, Mogensen J, Kristiansen K, Bolund L and Gregersen N: Genomic structure of the human mitochondrial chaperonin genes: HSP60 and HSP10 are localised head to head on chromosome 2 separated by a bidirectional promoter. Hum Genet 112: 71-77, 2003.

23. Cao Z, Ma J and Yuan WJ: Heat shock protein 60 in cell apoptosis. Sheng Li Ke Xue Jin Zhan 39: 267-270, 2008 (In Chinese).

24. Gupta S and Knowlton AA: HSP60 trafficking in adult cardiac myocytes: Role of the exosomal pathway. Am J Physiol Heart Circ Physiol 292: H3052-H3056, 2007.

25. Burguillos MA, Deierborg T, Kavanagh E, Persson A, Hajji N, Garcia-Quintanilla A, Cano J, Brundin P, Englund E, Venero JL and Joseph B: Caspase signalling controls microglia activation and neurotoxicity. Nature 472: 319-324, 2011.

26. Samali A, Cai J, Zhivotovsky B, Jones DP and Orrenius S: Presence of a pre-apoptotic complex of pro-caspase-3, Hsp60 and Hsp10 in the mitochondrial fraction of jurkat cells. EMBO J 18: 2040-2048, 1999.

27. Wang Y, Chen L, Hagiwara N and Knowlton AA: Regulation of heat shock protein 60 and 72 expression in the failing heart. J Mol Cell Cardiol 48: 360-366, 2010.

28. Rosenberger K, Dembny P, Derkow K, Engel O, Krüger C, Wolf SA, Kettenmann H, Schott E, Meisel A and Lehnardt S: Intrathecal heat shock protein 60 mediates neurodegeneration and demyelination in the CNS through a TLR-4 and MyD88-dependent pathway. Mol Neurodegener 10: 5, 2015.

29. Montgomery SL and Bowers WJ: Tumor necrosis factor-alpha and the roles it plays in homeostatic and degenerative processes within the central nervous system. J Neuroimmune Pharmacol 7: 42-59, 2012. 